\title{
Magnetic field generation during inflation
}

\author{
Chiara Caprini* \\ Institut de Physique Théorique \\ E-mail: chiara.capriniecea.fr
}

Large scale magnetic fields are ubiquitous in the universe: they are observed in galaxies and clusters, even at high redshift, and recently a lower bound on the magnetic field amplitude in the inter-galactic medium has been established using gamma ray telescopes. The origin of cosmological magnetic fields is still unknown, but their presence in the voids among structures points towards a generation in the very early universe. However, there is currently consensus about the absence of a satisfactory model, based on a gauge invariant lagrangian, able to produce during inflation the magnetic fields present in voids. After reviewing the considerations that lead to such conclusion, I will discuss how it is possible to evade them.

Frontiers of Fundamental Physics 14 - FFP14,

15-18 July 2014

Aix Marseille University (AMU) Saint-Charles Campus, Marseille

\footnotetext{
*Speaker.
} 